\title{
Article \\ Palatial Twistors from Quantum Inhomogeneous Conformal
Symmetries and Twistorial DSR Algebras $^{+}$
}

\author{
Jerzy Lukierski (D)
}

Citation: Lukierski, J. Palatial

Twistors from Quantum

Inhomogeneous Conformal

Symmetries and Twistorial DSR

Algebras. Symmetry 2021, 13, 1309.

https://doi.org/10.3390/sym13081309

Academic Editors: Ángel Ballesteros,

Giulia Gubitosi and Francisco J.

Herranz

Received: 3 May 2021

Accepted: 1 July 2021

Published: 21 July 2021

Publisher's Note: MDPI stays neutral with regard to jurisdictional claims in published maps and institutional affiliations.

Institute for Theoretical Physics, University of Wroclaw, Pl. Maxa Borna 9, 50-205 Wroclaw, Poland; jerzy.lukierski@ift.uni.wroc.pl

† Dedicated on 90-th Birthday to Sir Roger Penrose as a tribute to his ideas and achievements.

\begin{abstract}
We construct recently introduced palatial NC twistors by considering the pair of conjugated (Born-dual) twist-deformed $D=4$ quantum inhomogeneous conformal Hopf algebras $\mathcal{U}_{\theta}(s u(2,2) \ltimes$ $\left.T^{4}\right)$ and $\mathcal{U}_{\bar{\theta}}\left(s u(2,2) \ltimes \bar{T}^{4}\right)$, where $T^{4}$ describes complex twistor coordinates and $\bar{T}^{4}$ the conjugated dual twistor momenta. The palatial twistors are suitably chosen as the quantum-covariant modules (NC representations) of the introduced Born-dual Hopf algebras. Subsequently, we introduce the quantum deformations of $D=4$ Heisenberg-conformal algebra (HCA) $s u(2,2) \ltimes H_{\hbar}^{4,4}\left(H_{\hbar}^{4,4}=\bar{T}^{4} \ltimes_{\hbar} T_{4}\right.$ is the Heisenberg algebra of twistorial oscillators) providing in twistorial framework the basic covariant quantum elementary system. The class of algebras describing deformation of HCA with dimensionfull deformation parameter, linked with Planck length $\lambda_{p}$, is called the twistorial DSR (TDSR) algebra, following the terminology of DSR algebra in space-time framework. We describe the examples of TDSR algebra linked with Palatial twistors which are introduced by the Drinfeld twist and the quantization map in $H_{\hbar}^{4,4}$. We also introduce generalized quantum twistorial phase space by considering the Heisenberg double of Hopf algebra $\mathcal{U}_{\theta}\left(s u(2,2) \ltimes T^{4}\right)$.
\end{abstract}

Keywords: quantum deformations; quantum gravity; classical and quantum twistor geometry

\section{Introduction}

\subsection{Towards Quantum Gravity}

One can distinguish two basic levels in quantization procedure of physical models describing contemporary fundamental interactions:

(i) The first level can be called quantum-mechanical with canonically quantized phase space coordinates and possible presence of classical gravity only as a static background.

On such a level, we find all familiar relativistic quantum field theories, e.g., QED and QCD (fields quantized, space-time geometry flat and Minkowskian).

(ii) The second level also has quantized gravity and noncommutative space-times (all fields, including gravity and space-time geometry are quantized).

Quantum gravity (QG) remains a subject of rather hypothetical models (see, e.g., [1-4]), however it is mostly agreed that QG effects require at ultra-short distances the replacement of classical Einsteinian space-time by quantum noncommutative space-time geometry (see [5]). The QG-generated noncommutativity corrections appear as proportional to the powers of Planck mass $m_{p}$ or inverse powers of Planck length $\lambda_{p}$.

$$
\lambda_{p}=\frac{\hbar}{m_{p} c}=\sqrt{\frac{\hbar G}{c^{3}}}
$$

where $c$ is the light velocity and $G$ is the gravitational Newton constant. The QG origin of Planck length can be seen from Formula (1), with simultaneous presence of $\hbar$ and $G$.

In order to study algebraically the QG modifications of the space-time geometry in Special Relativity, one can look at the $\lambda$-dependent deformations $\mathcal{U}_{\lambda}\left(\mathcal{P}^{3,1}\right)$ of the Poincaré 
algebra $\mathcal{P}^{3,1}=o(3,1) \ltimes P^{3,1}$, where $P^{3,1}$ denotes the four-momenta sector and $\lambda$ describes an elementary length parameter which can be fixed $\lambda=\lambda_{p}$. Further, we consider the Minkowski space-time coordinates $x_{\mu} \in M^{3,1}$ together with covariantly acting Poincaré symmetry and introduce the semi-direct product algebra

$$
\mathbb{A}=\mathcal{P}^{3,1} \ltimes M^{3,1} \simeq o(3,1) \ltimes\left(P^{3,1} \ltimes M^{3,1}\right)
$$

where $P^{3,1} \ltimes M^{3,1}$ describes the relativistic phase space $\mathbb{P}^{3,1}=\left(M^{3,1} ; P^{3,1}\right)$, which after the first quantization level is endowed with relativistic Heisenberg algebra structure. Such algebra $\mathbb{A}_{\hbar}$, also called Heisenberg-Lorentz algebra, can be further deformed into quantum algebra $\mathcal{U}_{\lambda}\left(\mathbb{A}_{\hbar}\right)$, which, to describe quantum symmetry, should have Hopf algebra or Hopf-algebroid structure. We stress that only $U_{\lambda}\left(\mathcal{P}^{3 ; 1}\right)$ can be introduced as Hopf algebra, i.e., Drinfeld quantum symmetry group [6,7]. If $\hbar \neq 0$. the relativistic quantum phase space $\mathbb{P}_{\hbar}^{3 ; 1}$ implies that $U_{\lambda}\left(\mathbb{A}_{\hbar}\right)$ has the algebraic structure of Hopf algebroid [8-13].

Such class of deformations of algebra (2) provides so-called DSR (Doubly Special Relativity) algebra describing quantum space-times with covariantly acting quantum symmetry. We use the original name for DSR algebras [14-17], however some authors use the name DSR for "Deformed Special Relativity', which has a vague informative content.

The name "doubly" is due to the dependence on two parameters: $c$ (light velocity) and $\lambda_{p}$ (Planck length, or $m_{p} \sim\left(l_{p}\right)^{-1}$ ). The first parameter, $c$, appears in the physical basis of the relativistic classical algebra $\mathbb{A}$ and the second parameter, $\lambda$, determines the QG-induced modification of the algebraic structure (2).

It was argued already in the 1930s [18] that QG models should at the basic level depend on three fundamental nonvanishing constants, $c, \hbar$ and $G$, where $G$ can be replaced by $\lambda_{p}$ or $m_{p}$ (see (1)); if the cosmological constant or de Sitter radius of the Universe is finite, it introduces additional geometric parameter. The variant of DSR algebra with additional de Sitter radius as additional geometric parameter was called Triply Special Relativity (TSR); see [19].

The model of quantum space-time symmetries, which was an inspiration for introducing DSR algebras, is provided by the $\kappa$-deformed Poincaré-Hopf algebra $[20,21]$ with semi-direct product structure presented in [22] in so-called bicrossproduct basis.

Our aim is to describe some class of quantum-deformed twistors and provide the counterpart of DSR algebra in the noncommutative framework of quantum twistors. It should be recognized here that there are already several interesting papers dealing with quantum deformations of twistors and their geometries (see, e.g., [23-29]).

\subsection{Elements of Twistor Theory}

For more than half of a century, Roger Penrose and his collaborators (see, e.g., [23,30-32]) have promoted the idea that the space-time manifold is a secondary geometric construction, and primary geometric objects are twistors. In $D=4$ flat space-time, twistors are introduced as four-dimensional complex conformal spinors $t_{A} \in T^{4}$, endowed with the $D=4$ conformal-invariant pseudo-Hermitian $U(2,2)$ scalar product

$$
(\bar{t}, t) \equiv \bar{t}_{A} \eta^{A B} t_{B}, \quad A, B=1,2,3,4
$$

where $\bar{t}_{A} \in \bar{T}^{4}$ are the complex-conjugated dual twistors and $\eta^{A B}=(1,1,-1,-1)$. If we introduce twistors as the pair of $D=4$ Weyl spinors $(\alpha=1,2)$

$$
t_{A}=\left(\pi_{\alpha}, \omega^{\dot{\alpha}}\right)
$$

the alternative $U(2,2)$ frame should be used, with the metric

$$
\eta^{A B}=\left(\begin{array}{cc}
0 & 1_{2} \\
1_{2} & 0
\end{array}\right)
$$


leading to the formula

$$
(\bar{t}, t) \equiv \bar{t}_{A} \eta^{A B} t_{B}=\bar{\pi}_{\dot{\alpha}} \omega^{\dot{\alpha}}+\text { H.C. }
$$

We recall that the points $z_{\mu}$ of complex Minkowski space-time are specified in $T^{4}$ by two-dimensional planes with twistor coordinates, $\left(\pi_{\alpha}, \omega^{\dot{\alpha}}\right)$, satisfying the Cartan-Penrose incidence relation

$$
\omega^{\dot{\alpha}}=i z^{\dot{\alpha} \beta} \pi_{\beta}, \quad z^{\dot{\alpha} \beta}=\frac{1}{2}\left(\sigma^{\mu}\right)^{\dot{\alpha} \beta} z_{\mu} .
$$

The quantum-mechanical twistors $\hat{t}_{A}, \hat{t}_{A}$ on first basic quantization level are provided by the oscillator-like canonical commutation relations (CCR) [23,30].

$$
\begin{aligned}
& {\left[\hat{\bar{t}}_{A}, \hat{t}_{B}\right]=\hbar \eta_{A B}} \\
& {\left[\hat{t}_{A}, \hat{t}_{B}\right]=\left[\hat{t}_{A}, \hat{t}_{B}\right]=0 .}
\end{aligned}
$$

One can call $\hat{t}_{A}$ the twistor coordinates and $\hat{t}_{A}$ the twistor momenta; they introduce the twistorial analog of the relativistic quantum-mechanical phase-space algebra for conformalcovariant twistorial models. We recall that one can obtain the twistor realization of $D=4$ conformal algebra $o(4,2) \simeq s u(2,2)$ given by the bilinear products of Quantum-Mechanical (QM) twistors $\hat{t}_{A}, \hat{t}_{A}$ (see also Section 3.1).

The twistors $t_{A} \in T^{4}$ satisfying the Cartan-Penrose incidence relations (7) provide the geometric alternative for the description by complex Minkowski space-time geometry. The real Minkowski coordinates $x_{\mu}\left(z_{\mu}=x_{\mu}+i y_{\mu}\right)$ are obtained if the $2 \times 2$ Hermitian matrix $z^{\dot{\alpha} \beta}$ is parameterized as follows

$$
z^{\dot{\alpha} \beta}=\left(\begin{array}{cc}
x_{0}+x_{3} & x_{1}+i x_{2} \\
x_{1}-i x_{2} & x_{0}-x_{3}
\end{array}\right)=\left(z^{\dot{\beta} \alpha}\right)^{*}
$$

In such a case, one gets from (7) that $(t, t)=0$, i.e., the real Minkowski coordinates have as twistor counterparts the null twistor planes (so-called $\alpha$-planes), with vanishing norm (6).

For the discussion of QG effects, it is more appropriate and realistic to consider twistors corresponding to curved space-time. The simplest examples of nonflat space-times are the ones with constant curvature $R$ or cosmological constant $\Lambda= \pm \frac{1}{R^{2}}$, where $\Lambda>0$ for de Sitter and $\Lambda<0$ for anti-de Sitter geometries. In such a case, the standard quantization relations (8) and (9) for twistors should be modified, with respective deformation determined by the $\Lambda$-dependent antisymmetric constant second rank twistor $I_{A B}$, called in twistor theory the infinity twistor (see, e.g., [30]). In the basis (4), it is given by the following formula (we choose further $\Lambda>0$ )

$$
I_{A B}=\left(\begin{array}{cc}
\frac{\Lambda}{6} \epsilon_{\alpha \beta} & 0 \\
0 & \epsilon^{\dot{\alpha} \dot{\beta}}
\end{array}\right), \quad I_{A B}=-I_{B A} .
$$

One can introduce in twistorial phase space $\left(T^{4}, \bar{T}^{4}\right)$ the deformation of Poisson structure described by the following complex-holomorphic $(2,0)$ symplectic two-form [27]

$$
\Omega_{2}=d\left(I_{A B} t^{A} d t^{B}\right)=I_{A B} d t^{A} \wedge d t^{B}=\frac{\Lambda}{6} d \pi_{\alpha} \wedge d \pi^{\alpha}+d \omega_{\dot{\alpha}} \wedge d \omega^{\dot{\alpha}}
$$

generated by the holomorphic $(1,0)$ Liouville one-form $\Omega_{1}=I_{A B} t^{A} d t^{B}$, where $\Omega_{2}=d \Omega_{1}$. In dual twistor space $\bar{T}^{4}$, the complex-anti-holomorphic $(0,2)$ symplectic two-form (we denote $\bar{t}_{A} \equiv t_{A}^{\dagger}=\eta_{A B} \bar{t}^{B}$ )

$$
\bar{\Omega}_{2}=d\left(\bar{I}^{A B} \bar{t}_{A} d \bar{t}_{B}\right)=\bar{I}^{A B} d \bar{t}_{A} \wedge d \bar{t}_{B}=\frac{\Lambda}{6} d \bar{\pi}^{\dot{\alpha}} \wedge d \bar{\pi}_{\dot{\alpha}}+d \bar{\omega}^{\alpha} \wedge d \bar{\omega}_{\alpha}
$$


is complex-conjugated to (12), which leads to the relation

$$
\bar{I}^{A B}=\frac{1}{2} \epsilon^{A B C D} I_{C D}=\left(\begin{array}{cc}
\epsilon_{\alpha \beta} & 0 \\
0 & \frac{\Lambda}{6} \epsilon^{\dot{\alpha} \dot{\beta}}
\end{array}\right) .
$$

It appears that, within the framework of Hopf-algebraic quantum deformations of inhomogeneous conformal algebras $s u(2,2)$, one gets separately the deformations of twistors $\hat{t}^{A} \in T^{4}$ and $\hat{t} \in \bar{T}^{4}$, which lead to the quantum-mechanical (first level) quantization of symplectic structures (12) and (13).

The deformed twistors obtained by the quantization of symplectic Poisson structures (12)-(14) are called the palatial twistors [27]. After the quantization procedure, one gets the holomorphic and anti-holomorphic noncommutativity relations modifying (9) as follows (further, in many formulae, we put $\hbar=c=1$ ).

$$
\left[\hat{t}^{A}, \hat{t}^{B}\right]=\hbar \bar{I}^{A B}, \quad\left[\hat{\bar{t}}_{\dot{A}}, \hat{\bar{t}}_{\dot{B}}\right]=\hbar I_{\dot{A} \dot{B}}
$$

\subsection{Twist Deformations: From Space-Time to Twistors}

Let us recall the well-known twisting procedure (see, e.g., $[33,34])$ of the Poincare algebra $\mathcal{U}\left(o(3,1) \ltimes P^{3,1}\right)$ which is semi-dual to twisted Poincaré algebra $\mathcal{U}\left(o(3,1) \ltimes M^{3,1}\right)$.

For these two basic relativistic algebras (in Minkowski space-time and four-momentum space), one introduces the following basic Abelian twists

$$
\begin{array}{ll}
\bar{F}=\exp \left(i \frac{\lambda^{2}}{2} \theta^{\mu v} p_{\mu} \otimes p_{v}\right), & p_{\mu} \in P^{3,1} \\
F=\exp \left(i \frac{1}{2 \lambda^{2}} \theta_{\mu \nu} x^{\mu} \otimes x^{v}\right), & x^{\mu} \in M^{3,1}
\end{array}
$$

where $\theta_{\mu \nu}=-\theta_{v \mu}$ are real and dimensionless; the dimensionfull deformation parameter $\lambda$ representing an elementary length in QG applications may be chosen as Planck length $\lambda_{p}$. If we insert the twists (16) and (17) into the formulae for twist-deformed Hopf algebra modules (see, e.g., [5,35]), we get the quantization maps

$$
\hat{x}^{\mu}=m\left[\bar{F}^{-1}(\triangleright \otimes 1)\left(x^{\mu} \otimes 1\right)\right], \quad \hat{p}_{\mu}=m\left[F^{-1}(\triangleright \otimes 1)\left(p_{\mu} \otimes 1\right)\right],
$$

One obtains explicitly the well-known noncommutative $\theta$-deformed coordinates called also DFR (Dopplicher, Fredenhagen, Roberts). quantum space-times [36,37], where standard parameters $\theta_{D S R}^{\mu \nu}$ in DSR deformation are dimensionfull- $\theta_{D S R}^{\mu \nu}=\lambda^{2} \theta^{\mu \nu}$, with the dimensionality $\left[\theta_{D S R}^{\mu \nu}\right]=L^{2}$ and $\left[\theta^{\mu \nu}\right]=L^{0}$. The choice of $\theta^{\mu \nu}$ is purely geometric, selects directions in space-time, which are being deformed. We obtain from (18)

$$
\left[\hat{x}^{\mu}, \hat{x}^{\nu}\right]=i \lambda^{2} \theta^{\mu \nu}
$$

For the noncommutative $\theta$-deformed four-momenta we get

$$
\left[\hat{p}_{\mu}, \hat{p}_{\nu}\right]=\frac{i}{\lambda^{2}} \theta_{\mu \nu}
$$

If we wish to obtain both deformations (19) and (20) inside one algebraic structure, we observe that due to Jacobi identity the canonical relations $\left[x_{\mu}, p_{\nu}\right]=i \hbar g_{\mu \nu}$ should be modified and both deformations (19) and (20) can be embedded together only in a Hopf algebroid (see, e.g., [8-11]).

The paper is organized as follows. In Section 2, we describe the pair of twisted inhomogeneous $D=4$ conformal algebras $i s u(2,2) \equiv s u(2,2) \ltimes T^{4}, \bar{i} s u(2,2) \equiv s u(2,2) \ltimes$ $\bar{T}^{4}$. Using Hopf-algebraic twisting, we derive the NC relations (15) for holomorphic (chiral) and anti-holomorphic (anti-chiral) palatial twistors as the twist-generated quantum deformations. If we twist the primitive coproducts of $s u(2,2)$ generators, we can show that 
the twistorial NC phase space coordinates $\left(\hat{T}^{4}, \hat{T}^{4}\right)$ are covariant as Hopf algebra module under the action of respective twist-deformed inhomogeneous $s u(2,2)$ algebras.

In Section 3, we introduce the new notion of twistorial DSR (TDSR) algebra, in particular as $\Theta_{A B}$-deformed twistorial $D=4$ Heisenberg-conformal algebra $s u(2,2) \ltimes H_{\hbar}^{4,4}$, where $H_{\hbar}^{4,4}=\bar{T}^{4} \ltimes_{\hbar} T^{4}$ containing both sectors $T^{4}, \bar{T}^{4}$ simultaneously $\Theta$-deformed. For such purpose, we use the quantization map which follows from the quantized versions of symplectic structures (12) and (13), and suitably modifies the Kronecker delta in the relation (8). We propose the twist quantization by a Drinfeld twist $\mathbb{F}$ (see. e.g., [38]), which is related with the twists (29) and (30) and only in the linear approximation in $\lambda$ of the quantization map provides the palatial twistors. Further, we consider generalized twistorial quantum phase spaces defined by twisted Heisenberg doubles of $i s u(2,2)$ and $\bar{i} s u(2,2)$ Hopf algebras. In such a framework, following Brain and Majid, the quantum deformation of twistor geometry considered in Section 1.2 can be obtained by cotwist quantization of inhomogeneous conformal quantum groups $\operatorname{ISU}(2,2)$ and $\bar{I} S U(2,2)$, which, respectively, are Hopf-dual to twist-deformed isu $(2,2)$ and $i s u(2,2)$ quantum symmetry algebras.

In the concluding Section 4, we present an outlook, with directions for possible future research.

\section{Twisted Inhomogeneous $D=4$ Conformal Algebras, Born Duality and Palatial Twistors}

\subsection{From Poincaré to Inhomogeneous Conformal Algebras}

If we pass from the $D=4$ relativistic space-time description of the Universe to twistorial geometric framework, the $D=4$ Lorentz algebra $o(3,1) \simeq S L(2 ; \mathbb{C}) \oplus S L(2 ; \mathbb{C})$ with the four-vectors $x_{\mu}, p_{v}$ is replaced by $D=4$ conformal algebra $o(4,2) \simeq s u(2,2)$ with fundamental translational degrees of freedom described by twistors $\left(\hat{t}_{A} \in T^{4}, \hat{t}_{A} \in \bar{T}^{4}\right)$ spanning the canonical twistorial phase space. In the space-time approach, the orbital part of Lorentz algebra generators $M_{\mu \nu}$ can be realized in terms of $D=4$ relativistic phase space variables $\left(x_{\mu} \in M^{3,1}, p_{\mu} \in P^{3,1}\right)(\mu, v=0,1,2,3)$ as follows

$$
o(3,1): \quad M_{\mu v}=\hat{x}_{[\mu} \hat{p}_{v]} .
$$

where the Formula (21) is applicable only to the spinless systems; for the extension with spin in the context of quantum deformations, see, e.g., [13].

Using the twistorial canonical oscillator algebra (8) and (9), one can analogously express in terms of twistorial phase space coordinates $\left(\hat{t}_{A}, \hat{t}_{A}\right)$ the conformal generators $S_{A \bar{B}} \in \operatorname{su}(2,2)(A, B=1,2,3,4)$ (Using the basis (4) the physical 15 generators of $D=4$ conformal algebra can be expressed by the following bilinear formulas (see also Section 3.1)).

$$
\begin{array}{rr}
p_{\alpha \dot{\beta}}=\pi_{\alpha} \bar{\pi}_{\dot{\beta}} \quad M_{\alpha \beta}=\pi_{\alpha} \omega_{\beta} & M_{\dot{\alpha} \dot{\beta}}=\bar{\pi}_{\dot{\alpha}} \bar{\pi}_{\dot{\beta}} \\
D=\pi_{\alpha} \omega^{\dot{\alpha}}+\text { H.C. } & K_{\alpha \dot{\beta}}=\omega_{\alpha} \bar{\omega}_{\dot{\beta}} \\
S_{A B}=\hat{t}_{A} \hat{\bar{t}}_{B}-\frac{1}{4}(t, t) \eta_{A B} & \eta^{A B} S_{A B}=0
\end{array}
$$

where $S_{A B}$ are the $4 \times 4$ pseudo-Hermitian complex matrix $u(2,2)$ generators

$$
S_{A B}^{\dagger}=\eta_{A C} S_{C D} \eta_{D B}
$$

One can introduce the pair of Poincaré groups $\left(\mathcal{P}_{x}^{3,1}=O(3,1) \ltimes M^{3,1}, \mathcal{P}_{p}^{3,1}=\right.$ $\left.O(3,1) \ltimes P^{3,1}\right)$, with $M^{3,1}, P^{3,1}$ describing Minkowski coordinates and four-momenta, as two cosets related by the Fourier transform

$$
M^{3,1}=\frac{\mathcal{P}_{x}^{3,1}}{o(3,1)} \ni\left\{x_{\mu}\right\} \quad P^{3,1}=\frac{\mathcal{P}_{p}^{3,1}}{o(3,1)} \ni\left\{p_{\mu}\right\} .
$$


The twistorial counterparts of relations (24) appear as follows

$$
T^{4}=\frac{\operatorname{ISU}(2,2)}{\operatorname{SU}(2,2)} \quad \bar{T}^{4}=\frac{\bar{I} S U(2,2)}{\operatorname{SU}(2,2)}
$$

where $\operatorname{ISU}(2,2)=\operatorname{SU}(2,2) \ltimes T^{4}$ and $\bar{I} S U(2,2)=S U(2,2) \ltimes \bar{T}^{4}$ describe the semi-dual pair of twistorial inhomogeneous $D=4$ conformal groups. The twistors $t_{A} \in T^{4}$ describe the fundamental holomorphic conformal spinors (holomorphic twistors) and $\bar{t}_{A} \in \bar{T}^{4}$ the complex-conjugated anti-holomorphic ones. Alternatively, one can use the projective twistors $C P(3)$, described by the equivalence classes $t_{A} \sim c t_{A}(c \in \mathbb{C})$ in $T^{4}$, which parameterize the following symmetric coset of $S U(2,2)$ (see, e.g., [26]).

$$
C P(3)=\frac{S U(2,2)}{S(U(2,1) \otimes U(1))}
$$

The complex Minkowski coordinates $z^{\dot{\alpha} \beta} \in C M(4)$ (see (10)) can be introduced as parameterizing the following complex Grassmanian

$$
M(4)=\frac{S U(2,2)}{S(U(2) \otimes U(2))} .
$$

The coset (27) parameterizes complex 2-planes in $T^{4}$ which are determined by non-parallel pairs of intersecting twistors $t_{A}^{i}\left(i=1,2 ; A=1, \ldots 4 ;\left(t^{1}, t^{2}\right) \neq 0\right)$ and satisfy the pair (7) of Cartan-Penrose incidence relations. The complex Minkowski coordinates $z^{\mu}=\frac{1}{2}\left(\sigma^{\mu}\right) \dot{\alpha} \beta z^{\dot{\alpha} \beta}$ are expressed by the pair of intersecting twistor coordinates $t_{A}^{i}=\left(\pi_{\alpha}^{i}, \omega^{\dot{\alpha} i}\right)$ as follows

$$
z^{\dot{\alpha} \beta}=-\frac{i}{\pi^{1 \alpha} \pi_{\alpha}^{2}}\left(\omega^{1 \dot{\alpha}} \pi^{2 \beta}-\omega^{2 \dot{\alpha}} \pi^{1 \beta}\right) .
$$

The primary aim of the Penrose program during the last fifty years was to encode any curved Einsteinian space-time structure in geometric twistorial framework; in particular, it was important to find the vocabulary permitting to translate any general relativity solution in space-time into the twistorial language. This goal was however achieved only partially, with modest hopes that the program of finding the twistor formulation of general relativity theory will be fully successful. However, in last decade, Roger Penrose became inspired by the idea that perhaps it is an easier task to construct the twistorial noncommutative version of quantum gravity. Such a view, conceptually attractive, however still faces the basic question of how the appropriate formulation of quantum gravity in the space-time picture would look. On the twistorial side, some first steps towards the construction of twistorial quantum gravity model were provided by Penrose (see also [29]).

\subsection{Twist-Deformed Inhomogeneous Conformal Hopf Algebras and Holomorphic/ Anti-Holomorphic Quantum Twistors}

Our first task is to show how the relations (15) can be obtained in the framework of quantum deformations of inhomogeneous $D=4$ conformal algebras, with the respective holomorphic twistor coordinates $t_{A} \in T^{4}$ or anti-holomorphic twistorial complex momenta $\bar{t}_{A} \in \bar{T}^{4}$. For such a purpose, we consider the pair of semi-dual Hopf algebras $\mathbb{H}_{0} \equiv \mathcal{U}(i s u(2,2))=\mathcal{U}\left(s u(2,2) \ltimes T^{4}\right)$ and $\bar{H}_{0} \equiv \mathcal{U}(\bar{i} s u(2,2))=\mathcal{U}\left(s u(2,2) \ltimes \bar{T}^{4}\right)$, with Hermitian-conjugated generators in $T^{4}$ and $\bar{T}^{4}$, but with the same twistorial realization of $D=4$ conformal subalgebra $s u(2,2)$. Subsequently, one gets the holomorphic and anti-holomorphic palatial twistors if the Hopf algebras $\mathbb{H}_{0}$ and $\mathbb{H}_{0}$ are twisted, respectively by the following pair of twists, 


$$
\begin{aligned}
& \overline{\mathcal{F}}=\exp \left(-\frac{1}{2 \lambda} \bar{\Theta}^{A B} \hat{t}_{A} \wedge \hat{t}_{B}\right), \\
& \mathcal{F}=\exp \left(-\frac{1}{2 \lambda} \Theta_{A B} \hat{t}^{A} \wedge \hat{t}^{B}\right) .
\end{aligned}
$$

where further, in Section 3.1 we justify the same dependence of twists (29) and (30) from the elementary length parameter $\lambda$.

In the general case, the antisymmetric numerical tensor $\Theta_{A B}$ can be chosen as complex, but, in the case of Palatial twistors, because $\Theta_{A B}=I_{A B}$, they are real. The pair of twists (29) and (30) are dual under the twistorial Born map

$$
\hat{t}_{A} \longleftrightarrow \hat{t}_{A}, \quad \Theta_{A B} \rightarrow \bar{\Theta}_{A B}
$$

which, as the twistorial counterpart of the Born duality map $x_{\mu} \leftrightarrow p_{\mu}[39,40]$, interchanges the twistorial momenta $\hat{t}_{A}$ and the twistorial coordinates $\hat{t}_{A}$.

The conformal twists (29) and (30) deforming, respectively, isu(2,2) and isu $(2,2)$ Hopf-Lie algebras are the twistorial counterpart of the Poincare twists (16) and (17)-the second one employed quite often in the space-time approach (see $[33,34])$. Further, the pair of Formulae (18) has the following counterpart in twistorial description

$$
\begin{aligned}
& \hat{t}_{A}^{\overline{\mathcal{F}}}=m\left[\overline{\mathcal{F}}^{-1}(\triangleright \otimes 1)\left(\hat{t}_{A} \otimes 1\right)\right]=\left(\overline{\mathcal{F}}_{(1)}^{-1} \triangleright \hat{t}_{A}\right) \overline{\mathcal{F}}_{(2)}^{-1} \\
& \hat{t}_{A}^{\mathcal{F}}=m\left[\mathcal{F}^{-1}(\triangleright \otimes 1)\left(\hat{t}_{A} \otimes 1\right)\right]=\left(\mathcal{F}_{(1)}^{-1} \triangleright \hat{t}_{A}\right) \mathcal{F}_{(2)}^{-1} .
\end{aligned}
$$

The generators $\hat{t}_{A}^{\overline{\mathcal{F}}}$ describe the $\mathcal{U}_{\overline{\mathcal{F}}}(\bar{i} s u(2,2))$ Hopf algebra modules and $\hat{t}_{A}^{\mathcal{F}}$ span the module of Hopf algebra $\mathcal{U}_{\mathcal{F}}(i s u(2,2))$. Using the Hopf-algebraic action consistent with relation (6)

$$
\hat{\bar{t}}_{A} \triangleright \hat{t}_{B}=\eta_{A B} \quad \hat{t}_{A} \triangleright \hat{\bar{t}}_{B}=-\eta_{A B}
$$

one gets the following explicit formulae for conformal quantum twistors

$$
\begin{array}{ll}
\mathcal{U}_{\overline{\mathcal{F}}}(\bar{i} s u(2,2)): & \hat{t}_{A}^{\overline{\mathcal{F}}}=\hat{t}_{A}+\frac{1}{\lambda} \bar{\Theta}_{A}{ }^{B} \hat{\bar{t}}_{B} \\
\mathcal{U}_{\mathcal{F}}(\text { isu }(2,2)): & \hat{t}_{A}^{\mathcal{F}}=\hat{t}_{A}-\frac{1}{\lambda} \Theta_{A}{ }^{B} \hat{t}_{B} .
\end{array}
$$

Using the Formula (32) for $\bar{t}_{A}$ and (33) for $t_{A}$, one gets additionally

$$
\hat{t}_{A}^{\mathcal{F}}=\hat{t}_{A} \quad \hat{t}_{A}^{\overline{\mathcal{F}}}=\hat{t}_{A} .
$$

We obtain the following two algebras describing twist-deformed quantum twistorial phase space coordinates $\left(\hat{t}_{A}^{\mathcal{F}}, \hat{t}_{A}^{\mathcal{F}}\right)$ and $\left(\hat{t}_{A}^{\mathcal{F}}, \hat{t}_{A}^{\overline{\mathcal{F}}}\right)$

$$
\begin{array}{lll}
{\left[\hat{t}_{A}^{\mathcal{F}}, \hat{t}_{B}^{\mathcal{F}}\right]=\frac{2}{\lambda} \Theta_{A B}} & {\left[\hat{t}_{A}^{\mathcal{F}}, \hat{t}_{B}^{\mathcal{F}}\right]=0} & {\left[\hat{t}_{A}^{\mathcal{F}}, \hat{t}_{B}^{\mathcal{F}}\right]=\eta_{A B}} \\
{\left[\hat{t}_{A}^{\overline{\mathcal{F}}}, \hat{t}_{B}^{\overline{\mathcal{F}}_{B}}\right]=\frac{2}{\lambda} \bar{\Theta}_{A B}} & {\left[\hat{\hat{t}}_{A}^{\overline{\mathcal{F}}}, \hat{\bar{t}}_{B}^{\overline{\mathcal{F}}}\right]=0} & {\left[\hat{\bar{t}}_{A}^{\overline{\mathcal{F}}}, \hat{t}_{B}^{\overline{\mathcal{F}}}\right]=\eta_{A B} .}
\end{array}
$$

It should be stressed that the twistorial quantum phase space coordinates $\left(\hat{t}_{A}^{\bar{F}}, \hat{t}_{A}^{\mathcal{F}}\right)$ satisfy the following Hermitian-conjugated algebra

$$
\hat{t}_{A}^{\mathcal{F}}=\left(\hat{\bar{t}}_{A}^{\overline{\mathcal{F}}}\right)^{\dagger} \quad \hat{\hat{t}}_{A}^{\mathcal{F}}=\left(\hat{t}_{A}^{\overline{\mathcal{F}}}\right)^{\dagger} .
$$

The shifts described by Formulae (35) and (36) are the examples of the Bogolyubov transformation (see [41]) of twistorial oscillators satisfying the relations (8) and (9). 


\subsection{The Twisted Conformal Covariance of Quantum Twistors and Born Duality Map}

The relations (39) and (38) are quantum-covariant under the action of the twisted inhomogeneous conformal Hopf algebras $\mathbb{H}_{\mathcal{F}}=\mathcal{U}_{\mathcal{F}}(i s u(2,2))$ and $\mathbb{H}_{\overline{\mathcal{F}}}=\mathcal{U}_{\overline{\mathcal{F}}}(\bar{i} s u(2,2))$, respectively. In order to demonstrate such a property, one should calculate the twistdeformed coproducts using the familiar similarity maps

$$
\begin{array}{ll}
\Delta_{\mathcal{F}}(\hat{g})=\mathcal{F}^{-1} \circ \Delta_{0}(\hat{g}) \circ \mathcal{F} & \hat{g}=\left(\hat{t}, \hat{S}_{A \bar{B}}\right) \in i s u(2,2) \\
\Delta_{\overline{\mathcal{F}}}(\hat{g})=\overline{\mathcal{F}}^{-1} \circ \Delta_{0}(\hat{g}) \circ \overline{\mathcal{F}} & \hat{g}=\left(\hat{t}, \hat{S}_{A \bar{B}}\right) \in \bar{i} s u(2,2)
\end{array}
$$

where $\Delta_{0}(\hat{g})=\hat{g} \otimes 1+1 \otimes \hat{g}$ and the generators $\hat{S}_{A \bar{B}} \in s u(2,2)$ can be represented in terms of twistor coordinates $\left(\hat{t}_{A}, \hat{\vec{t}}_{A}\right)$ (see (21)). From Formulae (29), (30), (41) and (42), one obtains that

$$
\Delta_{\mathcal{F}}\left(\hat{t}_{A}\right)=\Delta_{0}\left(\hat{t}_{A}\right) \quad \Delta_{\overline{\mathcal{F}}}\left(\hat{t}_{A}\right)=\Delta_{0}\left(\hat{t}_{A}\right)
$$

and

$$
\begin{aligned}
& \Delta_{\mathcal{F}}\left(\hat{S}_{A \bar{B}}\right)=\Delta_{0}\left(\hat{S}_{A \bar{B}}\right)+\lambda^{-1}\left(\Theta_{B}^{D} \hat{t}_{A} \otimes \hat{t}_{D}+\Theta_{B}^{C} \hat{t}_{C} \otimes \hat{t}_{A}\right) \\
& \Delta_{\overline{\mathcal{F}}}\left(\hat{S}_{A \bar{B}}\right)=\Delta_{0}\left(\hat{S}_{A \bar{B}}\right)+\lambda^{-1}\left(\bar{\Theta}_{B}^{D} \hat{t}_{A} \otimes \hat{t}_{D}+\bar{\Theta}_{B}^{C} \hat{t}_{C} \otimes \hat{t}_{A}\right)
\end{aligned}
$$

Due to the modification using (44) and (45) of the primitive coproducts of $S_{A B}$, one can show that

$$
\begin{array}{ll}
\mathbb{H}_{\mathcal{F}}: & \hat{g} \triangleright\left(\left[\hat{\hat{t}}_{A}^{\mathcal{F}}, \hat{t}_{B}^{\mathcal{F}}\right]-\frac{2}{\lambda} \Theta_{A B}\right)=0 \\
\mathbb{H}_{\overline{\mathcal{F}}:} & \hat{\bar{g}} \triangleright\left(\left[\hat{\mathrm{t}}_{A}^{\overline{\mathcal{F}}}, \hat{\mathrm{t}}_{B}^{\overline{\mathcal{F}}}\right]-\frac{2}{\lambda} \bar{\Theta}_{A B}\right)=0
\end{array}
$$

where we use the standard Hopf-algebraic formula defining the action $\triangleright$ of generators $\hat{h} \in \mathbb{H}$ on the products $\hat{a} \cdot \hat{b}(\hat{a}, \hat{b} \in \mathbb{A})$

$$
\hat{h} \triangleright(\hat{a} \cdot \hat{b})=\left(\hat{h}_{(1)} \triangleright \hat{a}\right)\left(\hat{h}_{(2)} \triangleright \hat{b}\right) .
$$

where $\mathbb{A}$ is the $\mathbb{H}$-module algebra. We recall that, in the case of noncommutative $\theta_{\mu v^{-}}$ deformed quantum space-times and quantum four-momenta (see (19) and (20)), one gets analogously in coproducts $\Delta_{F}\left(M_{\mu v}\right)$ and $\Delta_{\bar{F}}\left(M_{\mu v}\right)$ the additional terms which are linear in $\theta_{\mu v}$ and bilinear in four-momenta (for twist (16)) or bilinear in space-time coordinates (for twist (17)); these terms are needed for the twisted quantum Poincare invariance of the algebraic relations (19) and (20) (see also [33]).

For coordinate and momenta twistors, one can consider the quantum covariance under two different inhomogeneous twisted conformal Hopf algebras $\mathcal{U}_{\mathcal{F}}(i s u(2,2))$ and $\mathcal{U}_{\overline{\mathcal{F}}}(\bar{i} s u(2,2))$, but they can be mapped into each other if we supplement the twistorial Born map (31) with the following exchange relation $\left(\Theta_{A B}\right.$ in the general case are complex, but it should be observed that $\theta_{\mu v}$ in both Formulae (19) and (20) is real and not changing under the map (49)).

$$
\Theta_{A B} \leftrightarrow \bar{\Theta}_{A B}
$$

The superposition of maps (31) and (49) leads to the following Born substitution rule of the twist factors (29) and (30)

$$
\mathcal{F} \leftrightarrow \overline{\mathcal{F}}
$$

The relations (49) and (50) describe the Born partial duality (semi-duality) of inhomogeneous conformal Hopf-algebras $\mathcal{U}_{\mathcal{F}}(i s u(2,2))$ and $\mathcal{U}_{\overline{\mathcal{F}}}(\overline{i s u}(2,2))$ (see, e.g., [42-44]) with interchanged subalgebras of twistorial momenta and coordinates.

\section{Twistorial DSR Algebra as Deformed Smashed Product of $s u(2,2)$ and Twistorial Quantum Phase Space}

\subsection{Twistorial DSR (TDSR) Algebra}

During 2000-2001, the notion of Double Special Relativity (DSR) was proposed, with the postulate that the geometry of special relativity in the presence of QG corrections is 
modified by quantum corrections, with Planck mass or Planck length playing the role of mass-like deformation parameter. In fact, in Snyder model [45], introducing first in the literature NC quantum space-time coordinates $\hat{x}_{\mu}$ by means of the relation

$$
\left[\hat{x}_{\mu}, \hat{x}_{v}\right]=g M_{\mu v} \quad[g]=L^{2}
$$

one usually assumes that $g=\beta l_{p}^{2}$ (where $\beta$ is a dimensionless constant) and one can consider Snyder model as the first historical example of DSR model. In the Hopf-algebraic framework of quantum groups, the general DSR algebra can be described as quantum algebra $\mathcal{U}_{\lambda}\left(\mathbb{A}_{D S R}\right)$, where $\mathbb{A}_{D S R}$ is given by the Formula (2).

If we wish to introduce the twistorial counterpart of DSR theory, described by the corresponding class of twistorial DSR algebras, one should replace the algebra $\mathbb{A}_{D S R}$ (see (2)) by the algebra $\mathbb{A}_{T D S R}$

$$
\mathbb{A}_{T D S R}=\bar{i} s u(2,2) \ltimes T^{4} \simeq i s u(2,2) \ltimes \bar{T}^{4} \simeq s u(2,2) \ltimes H_{\hbar}^{4,4}
$$

where $H_{\hbar}^{4,4}=\bar{T}^{4} \rtimes_{\hbar} T^{4}$ denotes the quantum twistorial phase space, described by the twistorial oscillators algebra (see (8) and (9)). Subsequently, one can introduce the twistorial DSR (TDSR) algebra as described by the following quantum deformations:

$$
\mathcal{U}_{\lambda}\left(\mathbb{A}_{T D S R}\right) \equiv \mathcal{U}_{\lambda}\left(s u(2,2) \ltimes_{\hbar} H_{\hbar}^{4,4}\right.
$$

In Formula (53), we use the particular case of semi-direct product, called smash product (see, e.g., [46,47]) of the Hopf algebra $\mathbb{H}$ and its module algebra $\mathbb{A}$

$$
\mathcal{H}=\mathbb{H} \ltimes \mathbb{A} .
$$

If $h, h^{\prime} \in \mathbb{H}$ and $a, b \in \mathbb{A}$, the multiplication rule in $\mathcal{H}$ is described by the following formula $\left(h \otimes a \in \mathcal{H} ; \Delta(h) \equiv h_{(1)} \otimes h_{(2)}\right)$

$$
(h \otimes a) \cdot\left(h^{\prime} \otimes b\right)=h \cdot h_{(1)}^{\prime} \otimes\left(a \triangleleft h_{(2)}^{\prime}\right) b
$$

which uses as input the coalgebraic sector in $\mathbb{H}$.

One can propose two ways of constructing TDSR algebra (53), in analogy with the two ways of describing the relativistic quantum NC phase space in Snyder model (see [48-50]):

(1) by proposing the quantum twistorial map as given by Formulae (35) and (36) (further in this section we link such a map with a cochain twist quantization);

(2) by calculating for the quantum Hopf algebras $\mathcal{U}_{\lambda}(i s u(2,2))$ and $\mathcal{U}_{\lambda}(\overline{i s} u(2,2))$ the Heisenberg double construction, which provides the generalized twistorial quantum phase space spanned by the quantum symmetry generators and the dual conformal quantum matrix group coordinates (for $\kappa$-deformed Poincaré- Heisenberg double see $[11,51]$; for $\theta_{\mu v}$-deformed Poincaré-Heisenberg double, see [52,53]).

\subsection{De Sitter Twistors and Length/Mass Dimensionalities}

In order to introduce into the twistor algebra in (8) and (9) the dimensionfull parameters, one can use the twistorial realization of conformal algebra generators $\left(P_{\mu}, M_{(\alpha \beta)}, M_{(\dot{\alpha} \dot{\beta})}\right.$, $\left.K_{\mu}, D\right)$, where

$$
\left[P_{\mu}\right]=L^{-1} \quad\left[M_{(\alpha \beta)}\right]=\left[M_{(\dot{\alpha} \dot{\beta})}\right]=[D]=L^{0}, \quad\left[K_{\mu}\right]=L
$$

describe the length dimensionalities $[L]=[M]^{-1}$ ([M] describes the mass dimensionality). Recalling twistorial realization of conformal algebra

$$
\begin{array}{r}
p_{\alpha \dot{\beta}}=\pi_{\alpha} \bar{\pi}_{\dot{\beta}} \quad M_{(\alpha \beta)}=\left(M_{(\dot{\alpha} \dot{\beta})}\right)^{\dagger}=\pi_{(\alpha} \omega_{\beta)} \\
D=\pi_{\alpha} \omega^{\alpha}+\text { H.C. } \quad K_{\alpha \dot{\beta}}=\omega_{\alpha} \bar{\omega}_{\dot{\beta}}
\end{array}
$$


we can easily deduce that the length dimensions of Weyl spinors $\left(\pi_{\alpha}, \omega_{\alpha}\right),\left(\bar{\pi}_{\dot{\alpha}}, \bar{\omega}_{\dot{\alpha}}\right)$ (see (4)) are the following

$$
\left[\pi_{\alpha}\right]=\left[\bar{\pi}_{\dot{\alpha}}\right]=L^{-\frac{1}{2}} \quad\left[\omega_{\alpha}\right]=\left[\bar{\omega}_{\dot{\alpha}}\right]=L^{\frac{1}{2}}
$$

In particular, one can introduce the rescaled dimensionless $\left([u]=[\bar{u}]=L^{0}\right)$ twistor components as follows

$$
u_{A}=\left(\begin{array}{c}
\lambda^{\frac{1}{2}} \pi_{\alpha} \\
\lambda^{-\frac{1}{2}} \bar{\omega}^{\dot{\alpha}}
\end{array}\right) \quad \bar{u}_{A}=\left(\begin{array}{c}
\lambda^{\frac{1}{2}} \bar{\pi}_{\dot{\alpha}} \\
\lambda^{-\frac{1}{2}} \omega^{\alpha}
\end{array}\right)
$$

where $\lambda$ is the fundamental length parameter. Generalizing the particular choice in (11) and (14) for palatial twistors to the antisymmetric tensorial matrix $\Theta_{A B}$

$$
\Theta_{A B}=\left(\begin{array}{cc}
\theta_{\alpha \gamma} & \theta_{\alpha \dot{\delta}} \\
\theta_{\dot{\beta} \gamma} & \theta_{\dot{\beta} \dot{\delta}}
\end{array}\right)
$$

we postulate the following dimensionalities

$$
\left[\theta_{\alpha \gamma}\right]=L^{2} \quad\left[\theta_{\alpha \dot{\delta}}\right]=\left[\theta_{\dot{\beta} \gamma}\right]=L \quad\left[\theta_{\dot{\beta} \dot{\delta}}\right]=L^{0} .
$$

consistent with the assignment $\Theta_{A B}=I_{A B}$.

Using (59) and (29)-(30), one obtains (recall the $[\lambda]=L$ ) that the expression

$$
\begin{gathered}
f \equiv-2 i \lambda \ln F=\Theta_{A B} \hat{t}^{A} \wedge \hat{t}^{B}= \\
\theta_{\alpha \gamma} \pi^{\alpha} \wedge \pi^{\gamma}+\theta_{\alpha \dot{\delta}} \pi^{\alpha} \wedge \bar{\omega}^{\dot{\delta}}+\theta_{\dot{\beta} \gamma} \bar{\omega}^{\dot{\beta}} \wedge \pi^{\gamma}+\theta_{\dot{\beta} \dot{\delta}} \bar{\omega}^{\dot{\beta}} \wedge \bar{\omega}^{\dot{\delta}}=\lambda \Theta_{A B}^{(0)} u^{A} \wedge u^{B}
\end{gathered}
$$

where $\theta_{\alpha \gamma}=\lambda^{2} \theta_{\alpha \gamma}^{(0)}, \theta_{\alpha \dot{\delta}}=\lambda \theta_{\alpha \dot{\delta}}^{(0)}, \theta_{\beta \dot{\delta}}=\theta_{\beta \dot{\delta}}^{(0)}$ and $\theta_{A B}^{90)}$ is dimensionless. It follows that $[f]=L$; similarly, one gets $[\bar{f}]=L$.

Due to these numerical values of dimensionalities in front of the exponent (63) in Formulae (29) and (30) the numerical factor $\lambda^{-1}$ appears. It should be observed that, contrary to the case of space-time twists (16) and (17), in both twistorial twists (29) and (30), the scaling normalization factor is the same.

\subsection{Twist Deformation of Twistors by Drinfeld Twist}

One can construct the Drinfeld twist (see, e.g., $[38,54,55])$ by multiplication of twococycle twists (29) and (30) in various ways, related by BCH-type formulas. Such twist can be used for twist quantization of the Heisenberg-conformal algebra (52), which becomes a quasi-bialgebroid described by the smash product of the conformal $s u(2,2)$ and canonical twistorial Heisenberg algebra as the $s u(2,2)$ module.

We consider the following cochain twist (see, e.g., [34]; $\lambda \sim \frac{1}{m_{p}}$ is real).

$$
\mathbb{F}=\exp \left[-\frac{1}{2 \lambda}\left(\Theta^{A B} \hat{t}_{A} \wedge \hat{t}_{B}+\bar{\Theta}^{A B} \hat{t}_{A} \wedge \hat{t}_{B}\right)\right]
$$

In Formula (64), the exponential factor is dimensionless. Because $\mathbb{F}$ does not satisfy the two-cocycle condition, the resulting twisted coproducts are not coassociative and the twist quantization will generate the quasi-bialgebroid structure.

One gets the twist-deformed quantum twistor variables $\hat{\xi}_{R}=\left(\hat{t}_{A}, \hat{\bar{t}}_{A}\right)(R=1 \cdots 8)$ as describing the twist quantization of Hopf algebra module

$$
\hat{\zeta}_{R}^{\mathbb{F}}=m\left(\mathbb{F}^{-1} \circ(\triangleright \otimes 1) \circ\left(\hat{\zeta}_{R} \otimes 1\right)\right) .
$$

If in (65), we insert (34) and (64), then calculate the contribution generated by the linear $\lambda$-term in $f=\ln \mathbb{F}$, we get 


$$
\begin{aligned}
& \hat{t}_{A}^{\mathbb{F}}=\hat{t}_{A}+\lambda^{-1} \bar{\Theta}_{A}^{B} \hat{\hat{t}}_{B}+o\left(\lambda^{-2}\right) \\
& \hat{t}_{A}^{\mathbb{F}}=\hat{t}_{A}-\lambda^{-1} \Theta_{A}^{B} \hat{t}_{B}+o\left(\lambda^{-2}\right)
\end{aligned}
$$

i.e., the linear term in the $\lambda^{-1}$ power expression gives the quantization maps analogous to (35) and (36). Taking only the linear term into consideration, we obtain the following modification of twistorial CCR (see (8) and (9))

$$
\begin{gathered}
{\left[\hat{t}_{A}^{\mathbb{F}}, \hat{t}_{B}^{\mathbb{F}}\right]=\left(\eta_{A B}-4 \lambda^{-2} \Theta_{A}^{C} \bar{\Theta}_{B C}\right)} \\
{\left[\hat{t}_{A}^{\mathbb{F}}, \hat{t}_{B}^{\mathbb{F}}\right]=2 \lambda^{-1} \bar{\Theta}_{A B} \quad\left[\hat{t}_{A}^{\mathbb{F}}, \hat{t}_{B}^{\mathbb{F}}\right]=2 \lambda^{-1} \Theta_{A B} .}
\end{gathered}
$$

The relations (66)-(69) without higher order terms in $\lambda^{-1}$ can be treated as describing the quantization map for $\Theta_{A B}$-deformed twistorial Heisenberg algebra.

The canonical conformal covariance relations for coordinate and momentum twistors $\left(A, B=1 \ldots 4 ; \hat{S}_{A B} \in \operatorname{su}(2,2)\right)$

$$
\begin{gathered}
{\left[\hat{S}_{A B}, \hat{t}_{C}\right]=\eta_{B C} \hat{t}_{A}} \\
{\left[\hat{S}_{A B}, \hat{t}_{\bar{C}}\right]=-\eta_{A C} \hat{t}_{\bar{B}}}
\end{gathered}
$$

after twisting by $\mathbb{F}$ do not remain valid. We arrive at higher order terms in Formulae (66) and (67) and in (70) and (71) additional terms which contain, besides the $s u(2,2)$ generators $S_{A B}$ (see (22)), the bilinear products $\hat{t}_{A} \hat{t}_{B}, \hat{t}_{A} \hat{t}_{B}$, which together form the set of generators of $S p(8 ; R)$ algebra, which is realized linearly on the eight-dimensional real twistor space $\rho_{R}=\left(\hat{t}_{A}+\hat{t}_{A}, i\left(\hat{t}_{A}-\hat{t}_{A}\right)\right.$. The algebra $S p(8 ; R)$ is known as providing the generalization of $D=4$ conformal symmetries in the presence of tensorial central charges [56], and it leads to numerous applications, e.g., in the Vasiliev higher spin algebras [57,58]. One can conclude therefore that the twist (64), which simultaneously deforms both the twistorial coordinates and momenta, could be better adjusted to the twist quantization of inhomogeneous generalized conformal algebra is $p(8 ; R)=R^{8} \rtimes S p(8 ; R)$.

We recall that, for the algebra (52) and its $\mathbb{F}$-twisted quantum version, the coalgebraic sector can be defined only in the framework of quasi-bialgebroids (quasi-Hopf algebroids) [8-13,59].

\subsection{Heisenberg Doubles and Generalized Twistorial Quantum Phase Space}

It can be shown that, in the $D=4$ space-time framework, both $(4+4)$-dimensional quantum phase space as well as the $(10+10)$-dimensional one which also contains the Lorentz sector can be described as the Heisenberg doubles, providing various generalizations and extensions of Heisenberg algebra.

The Heisenberg double is a special example of the smash product (54), when $\mathbb{A}$ is identified with the dual Hopf algebra $\mathbb{H}^{\star}$. In such a case, the nondegenerate bilinear Hopf pairing $\langle\cdot, \cdot\rangle: \mathbb{H} \otimes \mathbb{A} \rightarrow \mathbb{C}$ between two Hopf algebra $\mathbb{H}$ and $\mathbb{H}^{\star}$ is used, with the following action $\mathbb{H} \triangleright \mathbb{H}^{\star}$

$$
h \triangleright a=a_{(1)}<h, a_{(2)}>.
$$

Subsequently, one can write

$$
h \triangleright(a b)=a_{(1)}<h_{(1)}, a_{(2)}>b_{(1)}<h_{(2)}, b_{(2)}>=\left(h_{(1)} \triangleright a\right)\left(h_{(2)} \triangleright b\right)
$$

in accordance with the action (48) on the Hopf algebra module. One can derive in $\mathcal{H}$ the cross relations between the algebraic sectors of $\mathbb{H}$ and $\mathbb{H}^{\star}(h \equiv h \otimes 1, a \equiv 1 \otimes a)$

$$
h \cdot a=a_{(1)}<h_{(1)}, a_{(2)}>h_{(2)}
$$

which completes the multiplication table in $\mathbb{H} \otimes \mathbb{H}^{\star}$.

In applications of the Heisenberg double $\mathbb{H} \ltimes \mathbb{H}^{\star}$ to physical models, the Hopf-algebra $\mathbb{H}$ usually describes the generalized quantum momenta, while the dual Hopf algebra $\mathbb{H}^{\star}$ 
provides the sector of generalized quantum positions. In the four-dimensional spacetime approach, one obtains the generalized $(10+10)$-dimensional quantum phase space expressed as the Heisenberg double $\mathcal{H}^{(\mathcal{P})}(\mathbb{H}=\mathcal{U}(\hat{g})$ denotes the enveloping Hopf-Lie algebra and $\mathbb{H}^{\star}=\mathcal{C}(\mathbb{G})$ is the Hopf algebra of functions on the Lie group manifold $\mathbb{G}$ ):

$$
\mathcal{H}^{(\mathcal{P})}=\mathcal{U}(i \hat{o}(3,1)) \ltimes \mathcal{C}(I O(3,1))
$$

where $i \hat{o}(3,1)$ describes Poincaré algebra and $I O(3,1)$ the dual Poincaré group. In the quantum case, e.g., in applications to QG, both Hopf algebras in (75) can be quantumdeformed in a way preserving the Hopf-algebraic duality property, e.g., by twisting or $\kappa$-deformation $[11,51,52]$.

In the twistor approach, one can choose as the generalized twistorial quantum phase space the following Heisenberg double

$$
\mathcal{H}^{(\mathcal{T})}=\mathcal{U}_{\lambda}(i s u(2,2)) \ltimes \mathcal{C}_{\lambda}(\operatorname{ISU}(2,2))
$$

where the Planck length plays the role of dimensionfull deformation parameter. In particular, one can consider in (76) the twist deformations with twists $\mathcal{F}, \overline{\mathcal{F}}$ (see (29) and (30)). If we observe that the twistors $\hat{t}_{A}, \hat{t}_{A}$ as well as the twists (29) and (30) are related by the Born map (see (31)), we obtain the following table of four Hopf algebras, describing possible twist-deformed inhomogeneous $D=4$ quantum conformal symmetries and $D=4$ inhomogeneous quantum conformal groups

$$
\begin{array}{lcl}
\mathcal{U}_{\lambda}^{\mathcal{F}}(\text { isu }(2,2)) & \underset{\text { Hopf }}{\rightleftarrows} & \mathcal{C}_{\lambda}^{\mathcal{F}}(\bar{I} S U(2,2)) \\
\text { Born } \uparrow \text { duality } & & \text { Born } \uparrow \text { duality } \\
\mathcal{U}_{\lambda}^{\overline{\mathcal{F}}}(\bar{i} s u(2,2)) & \underset{\text { Hopf }}{\rightleftarrows} & \mathcal{C}_{\lambda}^{\overline{\mathcal{F}}}(\operatorname{ISU}(2,2)) .
\end{array}
$$

By Hopf duality, the twist quantization of the algebra $i s u(2,2)(i s u(2,2))$ is mapped into the cotwist quantization of the group $\operatorname{ISU}(2,2)(\bar{I} S U(2,2))$ with the following properties of algebraic and coalgebraic sectors

$$
\begin{aligned}
& \begin{array}{c}
\text { Twist quantization } \\
\text { of } \mathcal{U}(\text { isu }(2,2))
\end{array} \quad \leftarrow \stackrel{\text { Hopf }}{\text { duality }} \quad \begin{array}{c}
\text { cotwist quantization } \\
\text { of } \mathbb{C}(\operatorname{ISU}(2,2))
\end{array} \\
& \left(\begin{array}{c}
\text { multiplication in } \\
\text { algebra not changed, } \\
\text { coproducts modified }
\end{array}\right) \longleftrightarrow\left(\begin{array}{c}
\text { multiplication in } \\
\text { algebra modified, } \\
\text { coproducts unchanged }
\end{array}\right) \text {. }
\end{aligned}
$$

In $D=4$, the most general $(23+23)$-dimensional twistorial DSR (TDSR) algebra can be described by the deformed Heisenberg double (76) with $(4+4)$ NC degrees of freedom in $T^{4} \oplus \bar{T}^{4}$ and $(15+15)$-dimensional conformal sector as the subalgebra described by the $s u(2,2)$ Heisenberg double.

If the Hopf algebras $\mathcal{U}(i s u(2,2)), \mathcal{U}(i s u(2,2))$ are twist-deformed, the cotwist-deformed algebras $\mathcal{C}(\bar{I} S U(2,2)), \mathcal{C}(\operatorname{ISU}(2,2))$ provide noncommutative matrix entries of quantum $S U(2,2)$ group. The noncommutativity of matrix group elements $g_{B}^{A} \in S U(2,2)$ is determined by RTT relations (see, e.g., $[7,26]$ )

$$
R_{B D}^{A C} g_{E}^{B} g_{F}^{D}=g_{C}^{D} g_{B}^{A} R_{E F}^{B D}
$$

where the $R$-matrix is expressed by the following cotwist formula

$$
R_{B D}^{A C}=\left(\mathcal{F}^{T} \mathcal{F}^{-1}\right)_{B D}^{A C}
$$


The cotwist $\mathcal{F}_{B D}^{A C}$ dual to the twist $\mathbb{F}$ is determined by the following evaluation map:

$$
\mathcal{F}_{B D}^{A C}\left(g, g^{\prime}\right) \equiv<\mathbb{F} \mid g_{B}^{A} \otimes g_{D}^{\prime C}>.
$$

The noncommutative multiplication formula of cotwisted $\operatorname{SU}(2,2)$ matrix elements is given by the formula

$$
g \circ g^{\prime}=\mathcal{F}\left(g_{(1)}, g_{(1)}\right) \cdot g_{(2)} \cdot g_{(2)}^{\prime} \cdot \mathcal{F}^{-1}\left(g_{(3)}, g_{(3)}^{\prime}\right) .
$$

Because the twistor coordinates and momenta $T^{4}, \bar{T}^{4}$ as well as the complex Minkowski space-time coordinates are expressed by the element of $S U(2,2)$ group (see (25)-(27)), the NC multiplication rule (82) defines the noncommutativity of cotwist-deformed twistor coordinates $\hat{t}_{A}, \hat{\bar{t}}_{A}$ as well as the complex quantum Minkowski coordinates $\hat{z}^{\dot{\alpha} \beta}$. Further, using the cotwist-deformed multiplication rule (82), one can also derive the cotwist deformation of incidence relations (7).

\section{Outlook}

The aim of this paper is the presentation of some aspects of the NC framework for quantum-deformed twistors. Our inspiration came from the paper by Penrose [27] who under the name of palatial twistors introduced the "physical" class of $\Theta_{A B}$-deformed dS (de Sitter) twistors, with the parameters $\theta_{A B}$ determined geometrically by real de Sitter infinity twistor $I_{A B}$ (see (11)).

In our scheme, we reduce the multiparameter deformations effectively to the oneParametric ones by using the geometric degree of freedom which describes the variable Planck length or variable Planck mass. As an example, one can provide the generalized $\kappa$-deformations depending on the constant four-vector $a_{\mu}$, generating the following $a_{\mu^{-}}$ dependent quantum space-times [60]:

$$
\left[\hat{x}_{\mu}, \hat{x}_{v}\right]=i\left(a_{\mu} x_{\mu}-a_{\nu} x_{\mu}\right)
$$

where $a_{\mu}=\lambda a_{\mu}^{(0)}\left([\lambda]=L,\left[a_{\mu}^{(0)}\right]=L^{0}\right)$ and the fourvector $a_{\mu}^{(0)}$ are chosen as normalized, namely $\left(a_{\mu}^{(0)}\right)^{2}=-1$ for standard time-like $\kappa$-deformation, $a_{\mu}^{(0) 2}=1$ for tachyonic spacelike $\kappa$-deformation and $a_{\mu}^{(0) 2}=0$ for light cone $\kappa$-deformation.

The following are some directions in which one can continue the studies presented in this paper:

1. If we consider the twistor correspondence with complexified space-times, one should introduce the pair of dual twistors $\left(t_{A}, w_{A} ; t_{A} w^{A}=0\right)$ called ambitwistors, not linked by complex conjugation (Hermitian conjugation in quantized case), which provide the description of complex null geodesies in complexified Minkowski space $\mathbb{M}_{4}^{\mathbb{C}}$ [61-63]. In such a case, if $w^{A}=\left(\lambda^{\alpha}, \mu_{\dot{\alpha}}\right)$, one can introduce the symplectic 2 -form (see (13))

$$
\tilde{\Omega}_{2}=d\left(\tilde{I}^{A B} w_{A} d w_{B}\right)=\tilde{I}^{A B} d w_{A} \wedge d w_{B}=\frac{\lambda}{6} d \lambda_{\alpha} \wedge d \lambda^{\alpha}+d \mu^{\dot{\alpha}} \wedge d \mu_{\dot{\alpha}}
$$

where $\lambda=\frac{1}{r^{2}}$ appears as the second cosmological constant. In such a case, the curvatures $R$ and $r$ associated with twistors $t_{A}$ and $w_{A}$ can be different; in particular, if $R \gg r$, they may provide the tool to describe two de Sitter geometries characterizing the cosmological macroscopic distances and the ultrashort Planckian ones. It appears that the duality map $t_{A} \leftrightarrow w_{A}$, which implies the interchange relation $R \leftrightarrow r$, can be linked with Born duality relation (see, e.g., $[39,40,64,65])$. One can speculate that the presence of the pair of dual radii $(r, R)$ in ambitwistor framework can lead to the description of quantum effects simultaneously at ultrashort (radius $r$ ) and at macroscoping (radius R) cosmological distances.

2. The $D=4$ twistorial construction presented here can be quite easily generalized to $D=3$ and $D=6$ twistors, described by the $D=3$ and $D=6$ conformal groups $S p(4 ; \mathbb{R}) \simeq O(3,2)$ and $U_{\alpha}(4 ; \mathbb{H}) \simeq O(6,2)$. We add that the $D=4$ conformal group 
$\operatorname{SU}(2,2)$ can also be described as the antiunitary one $U_{\alpha}(4 ; \mathbb{C})[66,67]$. In such a way, we deal with the antiunitary family of groups $U_{\alpha}(4 ; \mathbb{F})$, where field $F=R, C, H$. In addition, since the 1970s, supertwistors [68] have been studied, which are a well recognized tool in the studies of superparticles, superstrings, supersymmetric gauge theories and supergravity.

3. Various quantum deformations of $\operatorname{SU}(2,2)$ and of its complexification $S L(4 ; \mathbb{C})$ have been used since the 1990s ([69-72]; see also [73]). One can recall that S. Zakrzewski, after classifying the $D=4$ Lorentz matrices [74], proposed the algebraic technique to classify the classical r-matrices of Poincare algebras [75] After providing the classical $\mathrm{SU}(2,2)$ r-matrices, it should be possible to obtain also the $r$-matrices for inhomogeneous (pseudo) unitary algebras.

4. Recently, the twistorial field-theoretic approach to formulate gauge theories and gravity in twistor space has been promoted (see, e.g., [76,77]), with the dynamics described by twistorial actions. By using local twistor geometry, one obtains in a natural way conformal gravity [78]; the twistorial model of Einstein gravity with non-zero cosmological constant can also be obtained by embedding into twistorial conformal gravity [76,77]. The formulation of QG in twistorial framework, by analogy with the approach presented in [5], may require as well the noncommutative twistorial quantum geometry.

Funding: The paper was supported by Polish National Science Center, project 2017/27/B/ST2/01902. Data Availability Statement: Not applicable.

Acknowledgments: The author would like to thank Mariusz Woronowicz for the collaboration at initial stage of this paper; Andrzej Borowiec and Mariusz Woronowicz for valuable discussions; and Evgenij Ivanov and Maciek Dunajski, the organizers, respectively, of Workshop SQ'19 (Erevan, August 2019) and the Conference “Loops meet twistors" (Marseille-Luminy, September 2019), where some of the results presented in this article have been presented.

Conflicts of Interest: The author declares no conflict of interest.

\section{References}

1. Oriti, D. (Ed.) Approaches to Quantum Gravity. In Collection of Papers; Cambridge University Press: Cambridge, UK, 2009.

2. Ashtekar, A.; Lewandowski, J. Background-independent quantum gravity: A status report. Class. Quant. Grav. 2004, 21, R53. [CrossRef]

3. Rovelli, C. Quantum Gravity; Cambridge University Press: Cambridge, UK, 2004.

4. Ambjorn, J.; Jurkiewicz, J.; Loll, R. Causal Dyamical Triangulations and the Quest for Quantum Gravity. In Foundations of Space-Time; Ellis, G., Murugen, J., Weltman, W., Eds.; Cambridge University Press: Cambridge, UK, 2012.

5. Beggs, E.; Majid, S. Quantum Riemannian Geometry; Serie in Mathematics; Springer: Berlin/Heidelberg, Germany, $2020 ;$ Volume 355.

6. Drinfeld, V.G. Quantum Groups. In Proceedings of the XXth Math. Congress, Berkeley, CA, USA, 3-11 August 1986; Berkeley Press: Berkeley, CA, USA, 1986; Volume 1, p. 798.

7. Majid, S. Foundations of Quantum Groups Theory; Cambridge University Press: Cambridge, UK, 2000.

8. Lu, J.-H. Hopf Algebroids and Quantum Grupoids. Int. J. Math. 1996, 7, 47-70. [CrossRef]

9. Brzezinski, T.; Militaru, G. Bialgebroids. $\times_{A}$-bialgebras and duality. J. Algebra 2002, 251, 279-294. [CrossRef]

10. Juric, T.; Kovacevic, D.; Meljanac, S. $\kappa$-deformed phase space, Hopf algebroid and twisting. SIGMA 2014, 10, 106. [CrossRef]

11. Lukierski, J.; Skoda, Z.; Woronowicz, M. $\kappa$-deformed covariant quantum phase spaces as Hopf algebroids. Phys. Lett. B 2015, 750, 401-406. [CrossRef]

12. Borowiec, A.; Pachol, A. Twisted bialgebroids versus bialgebroids from a Drinfeld twist. J. Phys. A Math. Theor. 2017, 50, 055205. [CrossRef]

13. Lukierski, J.; Meljanac, S.; Woronowicz, M. Quantum twist-deformed $D=4$ phase spaces with spin sector and Hopf algebroid structures. Phys. Lett. B 2019, 789, 82-87. [CrossRef]

14. Amelino-Camelia, G. Relativity in space-times with short-distance structure governed by an observer-independent (Planckian) length scale. Int. J. Mod. Phys. D 2002, 11, 35-59. [CrossRef]

15. Kowalski-Glikman, J. Observer-independent quanta of mass and length. Phys. Lett. A 2001, 286, 391-394. [CrossRef]

16. Kowalski-Glikman, J. Introduction to Doubly Special Relativity. In Planck Scale Effects in Astrophysics and Cosmology; Lecture Notes in Physics; Springer: Berlin/Heidelberg, Germany, 2005; Volume 669, pp. 131-159.

17. Borowiec, A.; Pachol, A. $\kappa$-Minkowski space-times and DSR algebras: Fresh look and old Problems. SIGMA 2010, 6, 86. [CrossRef]

18. Bronstein, M. Quantum theory of weak gravitational fields. JETP 1936, 9, 140157. 
19. Kowalski-Glikman, J.; Lee, S. Triply Special Relativity. Phys. Rev. D 2004, 70, 065020. [CrossRef]

20. Lukierski, J.; Ruegg, H.; Nowicki, A.; Tolstoy, V.N. q-deformation of Poincaré algebra. Phys. Lett. B 1991, 264, 331-338. [CrossRef]

21. Ballesteros, A.; Herranz, F.; del Olmo, M.; Santander, M. A new null-plane quantum Poincaré algebra. Phys. Lett. B 1995, 351, 137-145. [CrossRef]

22. Majid, S.; Ruegg, H. Bicrossproduct structure of $\kappa$-Poincaré group and non-commutative geometry. Phys. Lett. B 1994, 3-4, 348-354. [CrossRef]

23. Penrose, R. Twistor quantization and curved spacetime. Int. J. Theor. Phys. 1968, 1, 61-99. [CrossRef]

24. Kapustin, A.; Kuznetzov, A.; Orlov, D. Noncommutative instantons and twistor transforms. Comm. Math. Phys. 2001, 221, 385-432. [CrossRef]

25. Hannabus, K.C. Noncommutative twistor space. Lett. Math. Phys. 2001, 58, 153-166. [CrossRef]

26. Brain, S.; Majid, S. Quantization of twistor theory by cocycle twist. Commun. Math. Phys. 2008, 284, 713. [CrossRef]

27. Penrose, R. Palatial twistor theory and the twistor googly problem. Philos. Trans. R. Soc. A 2015, 373, 20140237. [CrossRef] [PubMed]

28. Lukierski, J.; Woronowicz, M. Noncommutative space-time from quantized twistors. In Proceedings of the Conference in Honour of 90th Birthday of Freeman Dyson, Singapore, 26-29 September 2013; Phua, K.K., Kwek, L.C., Chang, N.P., Chan A.H., Eds.; World Scientific: Singapore, 2014; p. 407.

29. Mercalli, M.; Penrose, R. Gluing noncommutative twistor space. Q. J. Math. 2021, 72, 417-454. [CrossRef]

30. Penrose, R.; MacCallum, M.A.H. Twistor theory: An Approach to the quantization of fields and space-time. Phys. Rep. 1972, 6, 241-315. [CrossRef]

31. Penrose, R. Nonlinear Gravitons and Curved Twistor Theory. Gen. Relativ. Gravit. 1976, 7, 31-52. [CrossRef]

32. Atiyah, M.; Dunajski, M.; Mason, L. Twistor theory at fifty: From contour integrals to twistor strings. Proc. R. Soc. Lond. A 2017, 473, 20170530. [CrossRef]

33. Chaichian, M.; Kulish, P.P.; Nishijima, K.; Tureanu, A. On a Lorentz-invariant interpretation of noncommutative space-time and its implications on noncommutative QFT. Phys. Lett. B 2004, 604, 98-102. [CrossRef]

34. Aschieri, P.; Lizzi, F.; Vitale, P. Twisting all the way: From Classical Mechanics to Quantum Fields. Phys. Rev. D 2008, 77, 025037. [CrossRef]

35. Aschieri, P.; Borowiec, A.; Pachol, A. Observables and dispersion relations in $\kappa$-Minkowski spacetime. JHEP 2017, 10, 152. [CrossRef]

36. Doplicher, S.; Fredenhagen, K.; Roberts, J. Space-time quantization induced by classical gravity. Phys. Lett. B 1994, 331, 39-44. [CrossRef]

37. Doplicher, S.; Fredenhagen, K.; Roberts, J. The quantum structure of spacetime at the Planck scale and quantum fields. Commun. Math. Phys. 1995, 172, 187-220. [CrossRef]

38. Drinfeld, V.G. Quasi-Hopf algebras. Algebra i Anal. 1989, 1, 114. (In Russian)

39. Born, M. Reciprocity theory of elementary particles. Rev. Mod. Phys. 1949, 21, 463. [CrossRef]

40. Freidel, L.; Kowalski-Glikman, J.; Leigh, R.G.; Minic, D. The Theory of metaparticles. Phys. Rev. D 2019, 99, 066011. [CrossRef]

41. Bogolyubov, N.N. On a new method in the theory of superconductivity. Nuovo Cim. 1958, 7, 794-805. [CrossRef]

42. Majid, S.; Schroers, B.J. q-Deformation and Semidualisation in 3d Quantum Gravity. J. Phys. A 2009, 42, 425402. [CrossRef]

43. Osei, P.K.; Schroers, B.J. On the semiduals of local isometry groups in 3rd gravity. J. Math. Phys. 2012, 53, 073510. [CrossRef]

44. Osei, P.K.; Schroers, B.J. Classical r-matrices via selfdualisation. J. Math. Phys. 2013, 54, 101702. [CrossRef]

45. Snyder, H.S. Quantized space-time. Phys. Rev. 1947, 71, 38. [CrossRef]

46. Klimyk, A.; Schmudgen, K. Quantum Groups and Their Representations; Springer: Berlin, Germany, 1997.

47. Blattner, R.; Cohen, M.; Montgomery, S. Crossed products and inner actions of Hopf algebras. Trans. Am. Math. Soc. 1986, 298, 671-711. [CrossRef]

48. Girelli, F.; Livine, E.R. Scalar field theory in Snyder space-time: Algernatives. JHEP 2011, 1103, 1-31. [CrossRef]

49. Meljanac, S.; Pachol, A. Heisenberg doubles for Snyder type models. arXiv 2021, arXiv:2101.02512.

50. Meljanac, S.; Mignemi, S. Unification of $\kappa$-Minkowski and extended Snyder spaces. Phys. Lett. B 2021, 814,136117 [CrossRef]

51. Lukierski, J.; Nowicki, A. Heisenberg double description of $\kappa$-Poincaré algebra and $\kappa$-deformed phase space. In Proceedings of the XXI International Colloquium on Group Theoretical Methods in Physics, Goslar, Germany, 15-20 July 1996; Dobrev, V.K., Doebner, H.D., Eds.; Heron Press: Sofia, Bulgaria, 1997; p. 186.

52. Borowiec, A.; Pachol, A. Heisenberg Doubles of Quantized Poincaré algebras. Theor. Math. Phys. 2011, 169, 1620-1628. [CrossRef]

53. Lukierski, J.; Woronowicz, M. Two $\theta_{\mu \nu}$-deformed covariant relativistic phase spaces as Poincaré -Hopf algebroids. Phys. Rev. D 2020, 101, 126003. [CrossRef]

54. Beggs, E.; Majid, S. Nonassociative Riemann geometry by twisting. J. Phys. Conf. Ser. 2010, 254, 012002; [CrossRef]

55. Beggs, E.J.; Majid, S. Quantizaton by cochain twists and nonassociative differentials. J. Math. Phys. 2010, 51, 05322 [CrossRef]

56. Bandos, I.; Lukierski, J.; Sorokin, D. Superparticle models with tensorial central charges. Phys. Rev. D 2000, 61, 045002 [CrossRef]

57. Vasiliev, M.A. Higher-Spin Theories and $S p(2 M)$ Invariant Space-Time. In Proceedings of the 3rd Sakharov Conference, Moscow, Russia, 24-29 June 2002.

58. Vasiliev, M.A. Higher Spin Superalgebras in any Dimension and their Representations. JHEP 2004, 0412,046 [CrossRef] 
59. Bulacu, D.; Panaite, F.; Van Oystaeyen, F. Quasi-Hopf algebra actions and smash products. Commun. Algebra 2000, 28, 631-651. [CrossRef]

60. Kosiński, P.; Maślanka, P. The kappa-Weyl group and its algebra. In From Quantum Field Theory to Quantum Groups; Jancewicz, B., Sobczyk, J., Eds.; World Scientific Publishing Company: Singapore, 2006; p. 41.

61. LeBrun, C. Spaces of complex null geodesics in complex Riemannian geometry. Trans. Am. Math. Soc. 1983, 278, 209-231. [CrossRef]

62. Geyer, Y.; Lipstein, A.E.; Mason, L.J. Ambitwistor Strings in Four Dimensions. Phys. Rev. Lett. 2014, 113, 081602 [CrossRef] [PubMed]

63. Baston, R.J.; Mason, L.J. Conformal Gravity, the Einstein Equations and Spaces of Complex Null Geodesics. Class. Quant. Grav. 1987, 4, 815. [CrossRef]

64. Freidel, L.; Rudolph, F.J.; Svoboda, D. A Unique Connection for Born Geometry. Commun. Math. Phys. 2019, 372, 119-150. [CrossRef]

65. Govaerts, J.; Jarvis, P.D.; Morgan, S.O.; Low, S.G. World-line quantisation of a reciprocally invariant system. J. Phys. A 2007, 40, 12095 [CrossRef]

66. Tits, J. Tabellen zu den Einfachen Lie Gruppen und Ihren Darstellungen; Lecture Notes in Mathematics; Springer: Berlin/Heidelberg, Germany, 1987; Volume 40.

67. Lukierski, J.; Nowicki, A. Quaternionic Supergroups and D = 4 Euclidean Extended Supersymmetries. Ann. Phys. 1986, 166, 164-188. [CrossRef]

68. Ferber, A. Supertwistors and conformal supersymmetry. Nucl. Phys. B 1978, 132, 55-64. [CrossRef]

69. Dobrev, V. Quantum deformations of noncompact Lie (super)algebras, Gőttingen Univ. preprint, July 1991. J. Phys. A 1993, 26, 1317. [CrossRef]

70. Lukierski, J.; Nowicki, A. Quantum deformations of $D=4$ Poincaré and Weyl algebra from $q$-deformed $D=4$ conformal algebra. Phys. Lett. B 1992, 279, 299-307. [CrossRef]

71. Lukierski, J.; Nowicki, A.; Sobczyk, J. All real forms of $U q(s l(4 ; C))$ and $D=4$ conformal quantum algebras. J. Phys. A 1993, 26, 4047. [CrossRef]

72. Frydryszak, A.; Lukierski, J.; Mozrzymas, M.; Minnaert, P. On quantum deformations of $D=4$ conformal algebra. In Proceedings of the VII-th Colloquium in Quantum Groups and Integrable Systems, Prague, Czech Republic, 18-20 June 1998. [CrossRef]

73. Tolstoy, V.N. Twisted quantum deformations of Lorentz and Poincaré algebra. Bulg. J. Phys. 2008, 35, 441-459.

74. Zakrzewski, S. Poisson structures on the Lorentz group. Lett. Math. Phys. 1994, 32, 11. [CrossRef]

75. Zakrzewski, S. Poisson structures on the Poincaré groups. Commun. Math. Phys. 1997, 185, 285-311. [CrossRef]

76. Adamo, T.; Mason, L. Conformal and Einstein Gravity from twistor action. Class. Quant. Grav. 2014, 31, 045014. [CrossRef]

77. Adamo, T. Twistor Actions for Gauge Theory and Gravity. Ph.D. Thesis, University of Oxford, Oxford, UK, 2013

78. Howe, P.S.; Lindström, U. Superconformal geometries and local twistors. arXiv 2020, arXiv:2012.03282. 